\title{
A molecular dynamics study of structure, stability and fragmentation patterns of sodium bis(2-ethylhexyl)sulfosuccinate positively charged aggregates in vacuo $\dagger$
}

\author{
Giovanna Longhi, ${ }^{* a b}$ Sergio Abbate, ${ }^{a b}$ Leopoldo Ceraulo, ${ }^{c d}$ Alberto Ceselli, ${ }^{e}$ \\ Sandro L. Fornili ${ }^{e}$ and Vincenzo Turco Liverif
}

Received 30th May 2011, Accepted 30th September 2011

DOI: $10.1039 / \mathrm{c1cp} 21740 \mathrm{~b}$

Positively charged supramolecular aggregates formed in vacuo by $n$ AOTNa (sodium bis(2-ethylhexyl)sulfosuccinate) molecules and $n_{\mathrm{c}}$ additional sodium ions, i.e. $\left[\mathrm{AOT}_{n} \mathrm{Na}_{n+n_{\mathrm{c}}}\right]^{n_{\mathrm{c}}}$, have been investigated by molecular dynamics (MD) simulations for $n=1-20$ and $n_{\mathrm{c}}=0-5$. Statistical analysis of physical quantities like gyration radii, atomic $B$-factors and moment of inertia tensors provides detailed information on their structural and dynamical properties. Even for $n_{\mathrm{c}}=5$, all stable aggregates show a reverse micelle-like structure with an internal solid-like core including sodium counterions and surfactant polar heads surrounded by an external layer consisting of the surfactant alkyl chains. Moreover, the aggregate shapes may be approximated by rather flat and elongated ellipsoids whose longer axis increases with $n$ and $n_{\mathrm{c}}$. The fragmentation patterns of a number of these aggregates have also been examined and have been found to markedly depend on the aggregate charge state. In one particular case, for which experimental findings are available in the literature, a good agreement is found with the present fragmentation data.

\section{Introduction}

Thanks to their typical chemical structure, surfactants are able to spontaneously self-assemble in the condensed phase and to form a large variety of organized aggregates: direct or reverse micelles, mono and multi-layers, admicelles, direct and reverse vesicles, water in oil and oil in water microemulsions, extended networks of micellar aggregates, organogels and liquid crystals. ${ }^{1-3}$ These aggregates are invariably characterized by the local positional and orientational order of surfactant molecules and by the coexistence of spatially separated hydrophilic and hydrophobic nanodomains. Such peculiar structural features

${ }^{a}$ Dipartimento di Scienze Biomediche e Biotecnologie,

Universita' di Brescia, Viale Europa 11, 25123 Brescia, Italy.

E-mail:longhi@med.unibs.it

${ }^{b}$ CNISM, Consorzio Interuniversitario Scienze Fisiche della Materia,

Via della Vasca Navale 84, 00146 Roma, Italy

${ }^{c}$ Dipartimento STeMBio, Via, Archirafi 32, 90123 Palermo, Italy

${ }^{d}$ Centro Grandi Apparecchiature, UniNetLAb, Via, F. Marini 14, 90128 Palermo, Italy

${ }^{e}$ Dipartimento di Tecnologie dell'Informazione, Università di Milano, Via, Bramante 65, 26013 Crema (CR), Italy

${ }^{f}$ Dipartimento di Chimica "S. Cannizzaro", Universita' degli Studi di Palermo, Viale delle Scienze Parco d'Orleans II, 90128 Palermo, Italy

$\dagger$ Electronic supplementary information (ESI) available: Fig. S1 and S2: dependence of $R_{\mathrm{GT}}$ and $R_{\mathrm{GC}}$ and of $a, b$, and $c$ from $n$ and $n_{\mathrm{c}}$. See DOI: $10.1039 / \mathrm{clcp} 21740 \mathrm{~b}$ find numerous technological applications, e.g. detergency, mineral flotation, bioprotection and food conservation, stabilization of molecular clusters and synthesis of nanocomposites.

Surfactants are also able to form aggregates in the gas phase. This was proven experimentally by analysing electrospray ionization (ESI) mass spectrometry data. The latter technique is particularly suitable to generate charged species without letting the surfactant molecules break, and to detect their mass and charge state. ${ }^{4-8}$ The preparation and characterization of aggregates with an aggregation number up to 554 surfactant molecules and charge state up to +18 was described in the literature, posing, as a consequence, fundamental questions about the spatial distribution of the excess charges within the aggregate and the effect on its size, shape and stability. ${ }^{9}$ It was shown experimentally that the maximum allowed charge state $\left(n_{\mathrm{c}, \max }\right)$ increases with the aggregation number $n \cdot{ }^{10,11}$

Additional information on the fragmentation patterns of charged surfactant aggregates was achieved by tandem mass spectrometry of the ions produced by isolating a selected precursor aggregate and collision induced dissociation (CID) with target gas. ${ }^{12}$ These spectra show that the fragmentation mechanism of singly charged surfactant aggregates consists in the loss of neutral species, while multiply charged species dissociate as couples of lower charge state aggregates. ${ }^{8,10}$

The value of the wealth of structural information from electrospray ionization mass spectrometry and tandem mass 
spectrometry of surfactants can be definitely enhanced by the results of computational methods that provide additional details on the structural organization of the aggregates, dynamical properties and dissociation pathways. For this reason we decided to carry out molecular dynamics (MD) simulations. In a previous MD study on neutral, positively and negatively singly charged aggregates of bis(2-ethylhexyl)sulfosuccinate $\left(\mathrm{AOT}^{-}\right.$) with $\mathrm{Na}^{+}, \mathrm{K}^{+}, \mathrm{Li}^{+}$and $\mathrm{Cs}^{+}$ions, ${ }^{13}$ we showed that these supramolecular species have a reverse micelle-like structure. Moreover, we recognized that the driving force of surfactant aggregation in the gas phase stems from the electrostatic interactions between surfactant head groups and counterions and that aggregates are elongated and rather flat ellipsoids.

Since the charge state is supposed to significantly impact onto the surfactant aggregate structure and stability, we were prompted to extend our previous investigation to consider multiply charged aggregates. Here we report some results obtained by MD simulation about the charge state effects on structural organization, stability and fragmentation patterns of positively charged aggregates formed in vacuo by $n \mathrm{AOTNa}$ (sodium bis(2-ethylhexyl)sulfosuccinate) molecules and $n_{\mathrm{c}}$ additional sodium ions, $\left[\mathrm{AOT}_{n} \mathrm{Na}_{n+n_{\mathrm{c}}}\right]^{n_{\mathrm{c}}}$, for $n=1-20$ and $n_{\mathrm{c}}=0-5$.

\section{Computational methods}

The model of the $\mathrm{AOT}^{-} R-R-R$ diastereoisomer (Fig. 1), reported here for ease of description, is based on the all-atom General Amber Force Field (GAFF), ${ }^{14}$ as previously described. ${ }^{13}$ In particular, the atomic charges of the $\mathrm{AOT}^{-}$ion were determined using the RESP protocol ${ }^{15}$ to comply with the AMBER force field, following quantum mechanical geometry optimization of a few molecular conformations at the RHF/ 6-31G* level. The $\mathrm{Na}^{+}$charge was assumed as $+1 e$. All simulated systems were prepared using the graphical version of the LeAP module of Amber Tools $1.4^{16}$ which enables the proper setting of mutual orientations and distances among the $\mathrm{AOT}^{-}$monomers and the placement of the $\mathrm{Na}^{+}$ions. Simulations were carried out using the AMBER $10^{16}$ SANDER module with a time step of $2 \mathrm{fs}$. The electrostatic interactions were evaluated by direct Coulomb sum. The SHAKE routine ${ }^{17}$

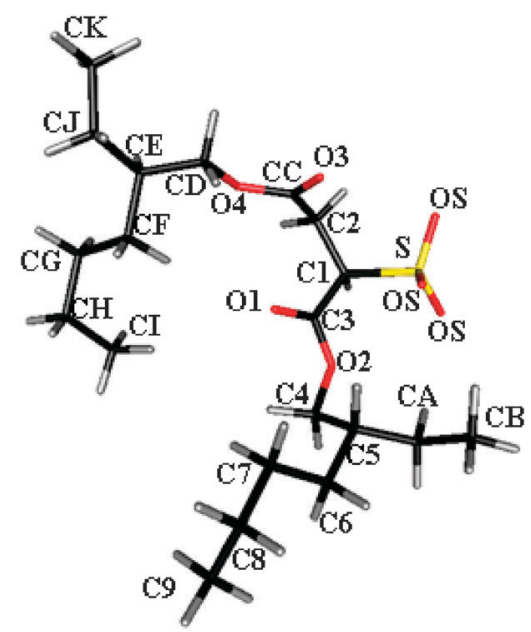

Fig. 1 Structure of the $\mathrm{AOT}^{-}$anion in the conformational minimum obtained by ab initio calculations. ${ }^{13}$

was used to constrain the bonds involving hydrogen atoms. The temperature was controlled according to the Berendsen coupling algorithm ${ }^{17}$ with 0.5 ps time constant and no cutoff was applied for non-bonded interactions (more precisely a $999 \AA$ cutoff was used). The equilibration phase of each simulation was performed according to the following protocol: in the first $1 \mathrm{~ns}$ time interval, the system self-aggregation at $300 \mathrm{~K}$ was induced by restraining the $\mathrm{AOT}^{-}-\mathrm{AOT}^{-}$and $\mathrm{AOT}^{-}-\mathrm{Na}^{+}$ intermolecular distances within $50 \AA$ by a flat-well potential function (i.e., no restraining force was applied until these distances were less than $50 \AA$, where a parabolic potential with a constant of $30 \mathrm{kcal} \mathrm{mol}^{-1} \AA^{-2}$ was switched on). This was found particularly useful for systems with high net charge, since the absence of a confining box and the reasonable but arbitrarily chosen and rather loose initial conformations made it difficult to obtain a single aggregate during this simulation phase. The system temperature was then gradually increased up to $600-700 \mathrm{~K}$ during the next $1 \mathrm{~ns}$ time interval, in order to make the system lose memory of the initial conformation and to speed up its evolution. The system temperature was then brought back to $300 \mathrm{~K}$ by gradually decreasing it in 100 40-ps steps, during which temperature was kept constant.

Table 1 Binding energy $E_{\mathrm{b}}$ for $\left[\mathrm{AOT}_{n} \mathrm{Na}_{n+n_{\mathrm{c}}}\right]^{n_{\mathrm{c}}}$ systems in vacuo as a function of the charge state $n_{\mathrm{c}}$, for various aggregation numbers $n$

\begin{tabular}{|c|c|c|c|c|c|c|c|c|c|c|}
\hline \multicolumn{11}{|c|}{$E_{\mathrm{b}} / \mathrm{kcal} \mathrm{mol}^{-1}$} \\
\hline$n_{\mathrm{c}}$ & $n=1$ & $n=2$ & $n=3$ & $n=4$ & $n=5$ & $n=6$ & $n=7$ & $n=8$ & $n=9$ & $n=10$ \\
\hline $\begin{array}{l}0 \\
1 \\
2 \\
3 \\
4 \\
5\end{array}$ & $\begin{array}{l}-121.9 \\
-176.3 \\
\text { Unstable }\end{array}$ & $\begin{array}{l}-307.9 \\
-362.1 \\
-350.2 \\
\text { Unstable }\end{array}$ & $\begin{array}{l}-481.9 \\
-559.7 \\
-548.8 \\
\text { Unstable }\end{array}$ & $\begin{array}{l}-681.4 \\
-751.1 \\
-751.5 \\
-693.6 \\
\text { Unstable }\end{array}$ & $\begin{array}{l}-863.8 \\
-937.2 \\
-942.6 \\
-900.0 \\
\text { Unstable }\end{array}$ & $\begin{array}{l}-1055.0 \\
-1131.5 \\
-1151.7 \\
-1102.3 \\
\text { Unstable }\end{array}$ & $\begin{array}{l}-1237.8 \\
-1311.0 \\
-1355.9 \\
-1326.6 \\
\text { Unstable }\end{array}$ & $\begin{array}{l}-1421.6 \\
-1507.2 \\
-1532.4 \\
-1496.5 \\
\text { Unstable }\end{array}$ & $\begin{array}{l}-1616.4 \\
-1694.0 \\
-1730.8 \\
-1702.7 \\
\text { Unstable }\end{array}$ & $\begin{array}{l}-1812.1 \\
-1895.1 \\
-1923.8 \\
-1903.3 \\
\text { Unstable }\end{array}$ \\
\hline$n_{\mathrm{c}}$ & $n=11$ & $n=12$ & $n=13$ & $n=14$ & $n=15$ & $n=16$ & $n=17$ & $n=18$ & $n=19$ & $n=20$ \\
\hline $\begin{array}{l}0 \\
1 \\
2 \\
3 \\
4 \\
5 \\
6\end{array}$ & $\begin{array}{l}-1991.3 \\
-2075.2 \\
-2106.6 \\
-2085.2 \\
\text { Unstable }\end{array}$ & $\begin{array}{l}-2178.6 \\
-2273.5 \\
-2312.0 \\
-2309.3 \\
\text { Unstable }\end{array}$ & $\begin{array}{l}-2375.6 \\
-2452.5 \\
-2504.7 \\
-2514.0 \\
-2432.6 \\
\text { Unstable }\end{array}$ & $\begin{array}{l}-2557.3 \\
-2636.3 \\
-2682.8 \\
-2676.3 \\
-2640.8 \\
\text { Unstable }\end{array}$ & $\begin{array}{l}-2746.7 \\
-2838.6 \\
-2888.8 \\
-2883.2 \\
-2823.7 \\
\text { Unstable }\end{array}$ & $\begin{array}{l}-2937.4 \\
-3014.6 \\
-3067.0 \\
-3061.3 \\
-3024.3 \\
\text { Unstable }\end{array}$ & $\begin{array}{l}-3130.3 \\
-3200.8 \\
-3243.6 \\
-3272.9 \\
-3224.5 \\
\text { Unstable }\end{array}$ & $\begin{array}{l}-3301.3 \\
-3382.1 \\
-3439.6 \\
-3457.2 \\
-3425.6 \\
\text { Unstable }\end{array}$ & $\begin{array}{l}-3495.8 \\
-3562.0 \\
-3675.4 \\
-3631.4 \\
-3642.4 \\
-3560.3 \\
\text { Unstable }\end{array}$ & $\begin{array}{l}-3710.7 \\
-3782.9 \\
-3840.0 \\
-3855.9 \\
-3851.3 \\
-3752.9 \\
\text { Unstable }\end{array}$ \\
\hline
\end{tabular}




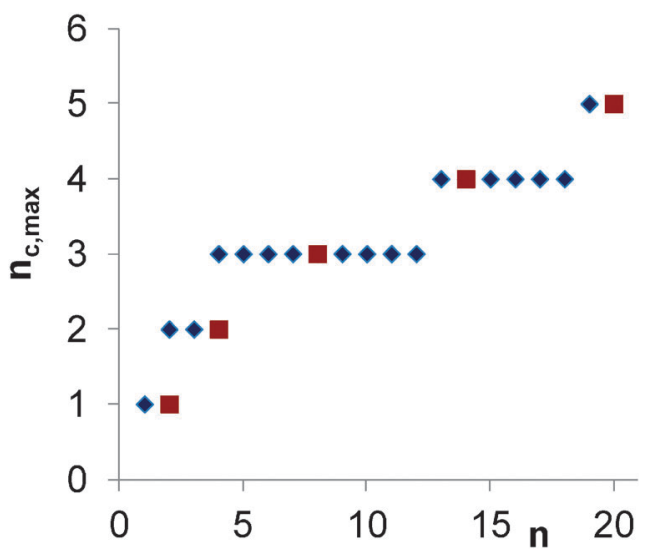

Fig. 2 Comparison of the dependence of maximum charge state $n_{\mathrm{c}, \max }$ upon the aggregate size $n$, as experimentally determined ( $\mathbf{\square}$, ref. 10) and evaluated by MD simulation ( $\diamond$, this work).

Constraints were then relieved and the simulations were continued at $300 \mathrm{~K}$ for $50 \mathrm{~ns}$.

To examine the fragmentation features of some aggregates obtained with this simulation procedure, twenty conformations were randomly chosen from the corresponding trajectory stretches stored during the last $1 \mathrm{~ns}$ interval. These conformations were assumed as initial conformations for further MD simulations during which the system temperature was increased in $4 \mathrm{~K}$ steps, each one lasting 40 ps. For each initial conformation, atom velocities were randomly generated according to a $300 \mathrm{~K}$ Maxwell distribution. Fragmentation events were recognized by direct inspection through graphical visualization of the trajectories. Eighty of such simulations were performed for the $\left[\mathrm{AOT}_{20} \mathrm{Na}_{20+3}\right]^{3+}$ system in order to compare more reliably the present fragmentation results with experimental data. ${ }^{10}$ The temperature at which fragmentation occurs may depend to some extent on the adopted computational procedure, thus we will discuss it only as an indication of the relative aggregate stability.

The statistical analysis of the trajectories was mainly based on the PTRAJ module of the Amber Tools 1.4 package, while the moments of inertia were calculated as previously described. ${ }^{13}$ Graphical analysis was performed using VMD, ${ }^{18} \mathrm{gOpenMol}^{19}$ and Rasmol. ${ }^{20}$

\section{Results and discussion}

Below we discuss the results of the statistical analysis of MD trajectories. We first concentrate on studying structure and stability of the aggregates, then we discuss the results of the MD fragmentation experiments.

\section{Structure and stability}

a. Analysis of interaction potential energy. In Table 1 we have collected the binding energy $\left(E_{\mathrm{b}}\right)$ values of the $\left[\mathrm{AOT}_{n} \mathrm{Na}_{n+n_{\mathrm{c}}}\right]^{n_{\mathrm{c}}}$ systems vs. the charge state $\left(n_{\mathrm{c}}\right)$ and the aggregation number $(n)$. These values are obtained by subtracting the potential energy of the isolated $\mathrm{AOT}^{-}$anions from that of the corresponding aggregate (i.e., $E_{\mathrm{b}}=E-n E_{\mathrm{AOT}^{-}}$). For comparison and completeness, in the following we consider also $n_{\mathrm{c}}=0$ cases. Each value has been obtained by averaging over the last $20 \mathrm{~ns}$ of the corresponding MD trajectory. From Table 1 one may notice that the binding energy progressively decreases with $n$, that instability occurs above a critical value of the charge state $n_{\mathrm{c}}$, which we call $n_{\mathrm{c}, \max }$, and that generally the binding energy starts to increase

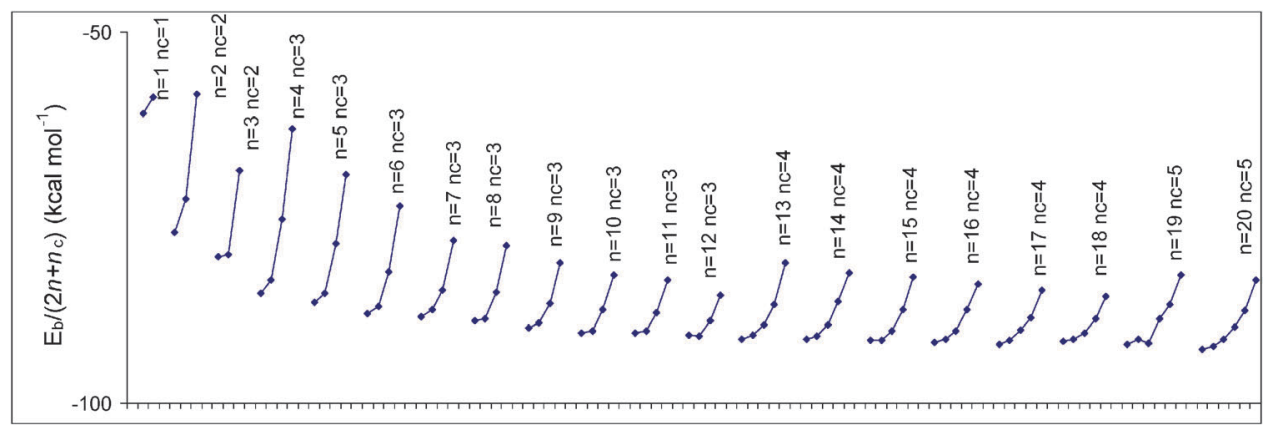

Fig. 3 Binding energy values per charged species, $\left(E_{\mathrm{b}} /\left(2 n+n_{\mathrm{c}}\right)\right)$, for $\left[\mathrm{AOT}_{n} \mathrm{Na}_{n+n_{\mathrm{c}}}\right]_{\mathrm{c}}^{n_{\mathrm{c}}}$ systems. Each continuous line refers to a single $n$ value and dots to different $n_{\mathrm{c}}$ values; only the cases with maximum charge excess, $n_{\mathrm{c}, \max }$, are labelled.

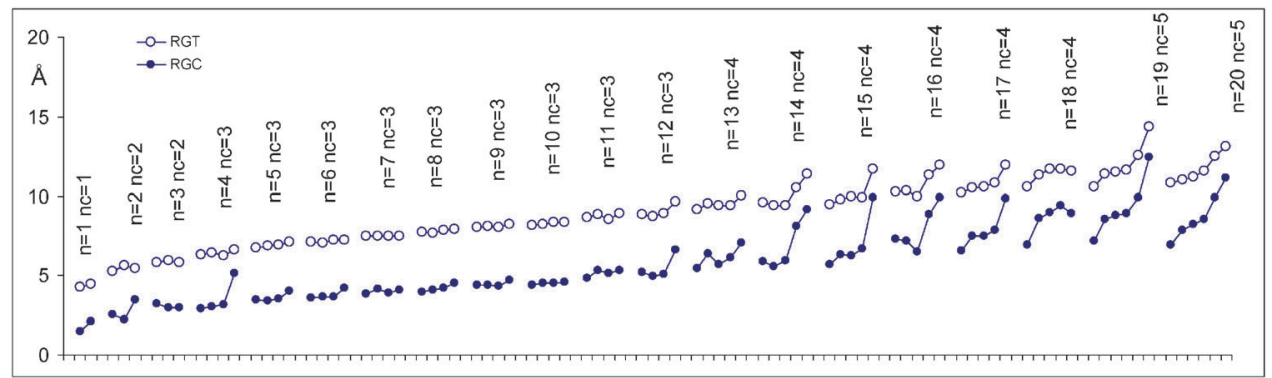

Fig. 4 Gyration radii $R_{\mathrm{G}}$ for $\left[\mathrm{AOT}_{n} \mathrm{Na}_{n+n_{\mathrm{c}}}{ }^{n_{\mathrm{c}}}\right.$ systems. Each continuous line refers to a single $n$ value and dots to different $n_{\mathrm{c}}$ values; only the cases with maximum charge excess, $n_{\mathrm{c}, \max }$, are labelled. Total gyration radius, $R_{\mathrm{GT}}$, empty circles; core gyration radius, $R_{\mathrm{GC}}$, full dots. 

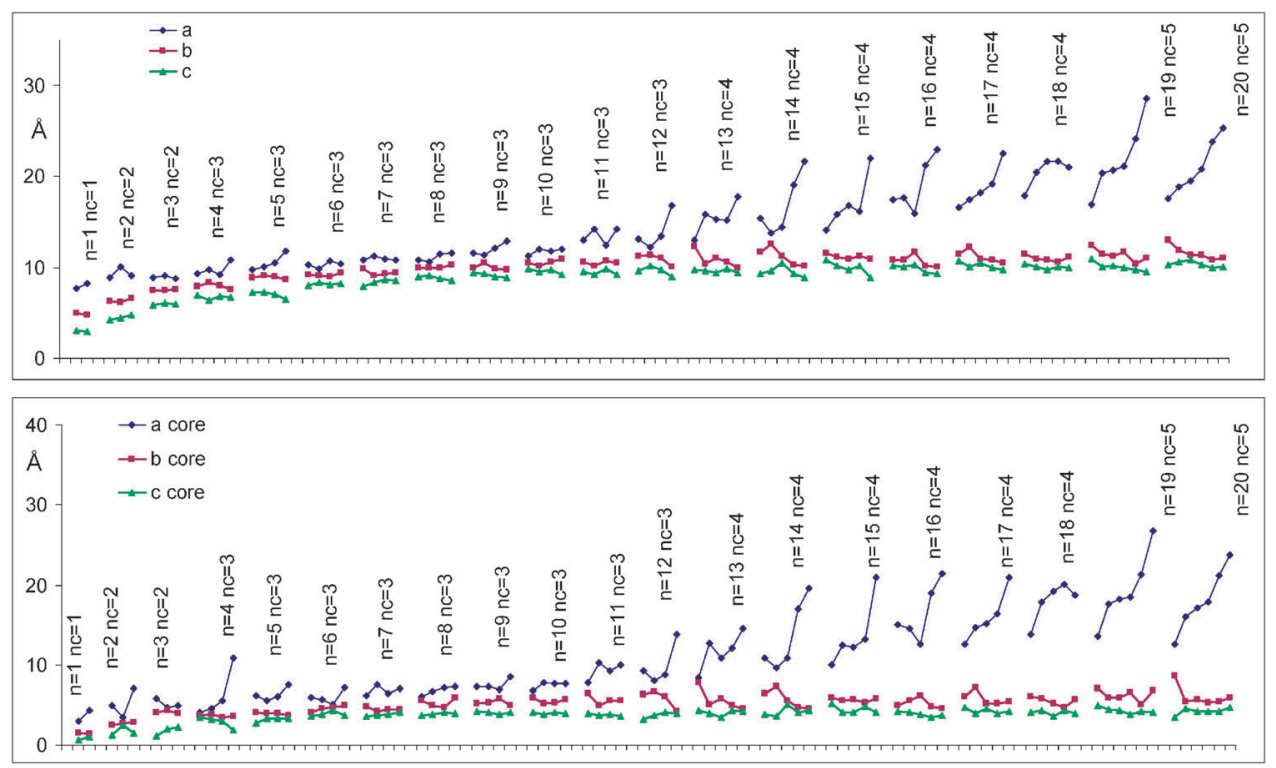

Fig. 5 Lengths of the semiaxes $a, b, c$ of $\left[\mathrm{AOT}_{n} \mathrm{Na}_{n+n_{\mathrm{c}}}\right]_{\mathrm{c}}^{n_{\mathrm{c}}}$ system shape approximated by ellipsoids (see text). Each continuous line refers to a single $n$ value and dots to different $n_{\mathrm{c}}$ values; only the cases with maximum charge excess, $n_{\mathrm{c}, \text { max }}$, are labelled.

at $n_{\mathrm{c}, \max }$. We also observe that addition of one AOTNa molecule to any $\left[\mathrm{AOT}_{n} \mathrm{Na}_{n+n_{\mathrm{c}}}\right]^{n_{\mathrm{c}}}$ aggregate, while keeping $n_{\mathrm{c}}$ constant, increases the stabilization energy of the aggregate by a nearly constant amount. Indeed, the binding energy $E_{\mathrm{b}}$ decreases linearly as $n$ increases, with a slope of about $195 \mathrm{kcal} \mathrm{mol}^{-1}$ (see also ref. 13).

The maximum excess charge values, $n_{\mathrm{c}, \max }$, vs. the aggregation number $n$ is shown in Fig. 2, where experimental data ${ }^{10}$ are also reported. This plot, which is stepwise since $n_{\mathrm{c}, \max }$ is an integer quantity, evidences the maximum excess charge bearable by an aggregate consisting of $n$ AOTNa molecules. One may notice that the experimental data agree fairly well with the simulation results and that, within each step, the capability to store additional charges increases with $n$ until the aggregate is able to safely accommodate one more sodium ion without fragmenting at $300 \mathrm{~K}$. Thus, e.g., a fourth excess charge cannot be safely stored on aggregates with $n$ less than 14 .

In Fig. 3 we report $E_{\mathrm{b}} /\left(2 n+n_{\mathrm{c}}\right)$ (namely, the binding energy values $E_{\mathrm{b}}$ for $\left[\mathrm{AOT}_{n} \mathrm{Na}_{n+n_{\mathrm{c}}}\right]^{n_{\mathrm{c}}}$ aggregates divided by the total number of charged particles $2 n+n_{\mathrm{c}}$, i.e., AOT $^{-}$plus $\mathrm{Na}^{+}$ ions) for the cases that we have examined ( $n=1-20$, $\left.n_{\mathrm{c}}=0-n_{\mathrm{c}, \max }\right)$. In this figure, each curve corresponds to a given value of $n$ and each dot to $n_{\mathrm{c}}=0-n_{\mathrm{c}, \max }$. We think that this type of plots allows one to appreciate finer details of the aggregate stability. In particular, it highlights that addition of extra charge increasingly but non-linearly destabilizes the aggregate. Moreover, an asymptotic value of about $-90 \mathrm{kcal} \mathrm{mol}^{-1}$ is attained by $E_{\mathrm{b}} /\left(2 n+n_{\mathrm{c}}\right)$ for neutral and single charged aggregates. The main contribution to the binding energy is electrostatic, as already observed in ref. 13, the total value resulting from a balance of the attractive interactions between negative polar heads and positive $\mathrm{Na}^{+}$ions and the repulsive interactions among negative polar heads and among positive ions.

b. Analysis of the shape of supramolecular aggregates. The charge state $n_{\mathrm{c}}$ has analogous effects on the shape both of the
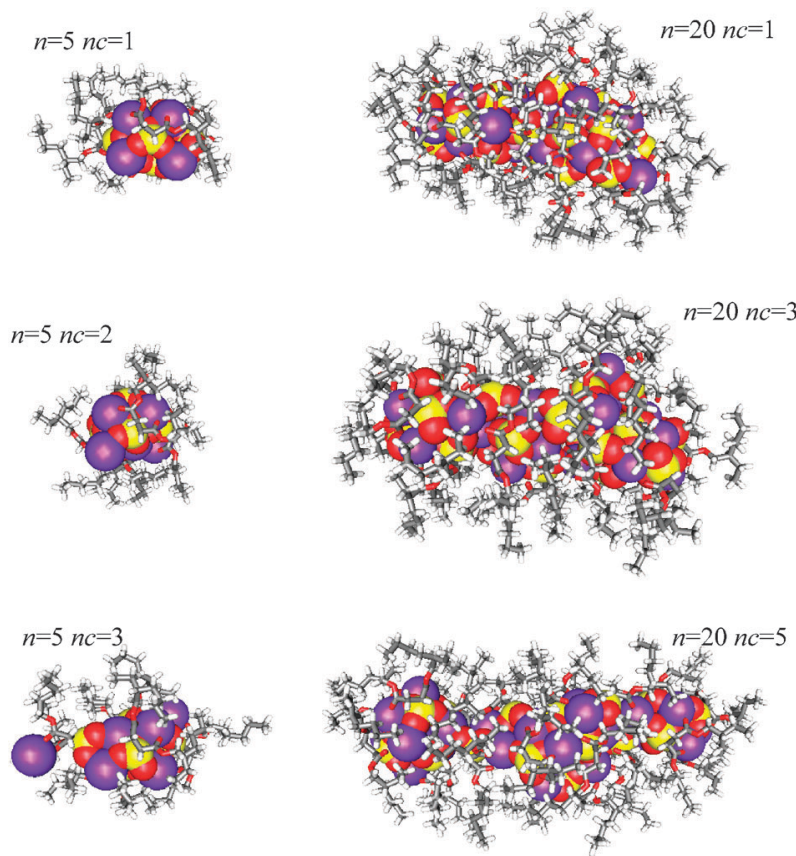

Fig. 6 Conformation of the $\left[\mathrm{AOT}_{5} \mathrm{Na}_{5+n_{\mathrm{c}}}\right]^{n_{\mathrm{c}}}, n_{\mathrm{c}}=1,2,3$ (left $)$ and $\left[\mathrm{AOT}_{20} \mathrm{Na}_{20+n_{\mathrm{c}}}\right]^{n_{\mathrm{c}}}, n_{\mathrm{c}}=1,3,5$ aggregates (right) as obtained by the MD simulation at $t=50 \mathrm{~ns}$. Sodium ions (blue) and $\mathrm{SO}_{3}{ }^{-}$polar heads (red oxygen atoms and yellow sulfur atoms) are displayed in a spacefilling mode to evidence structural properties of the micelle cores.

core and of the whole supramolecular aggregate. In order to examine how the aggregates change their shape as the excess positive charge increases, we plot geometrical parameters in a very similar way as we have done in Fig. 3. Let us first look at the mass weighted gyration radius $R_{\mathrm{G}}$ defined by the equation:

$$
R_{\mathrm{G}}=\sqrt{\frac{\sum_{i=1}^{N} m_{i}\left(\boldsymbol{r}_{i}-\boldsymbol{R}\right)^{2}}{\sum_{i=1}^{N} m_{i}}}
$$



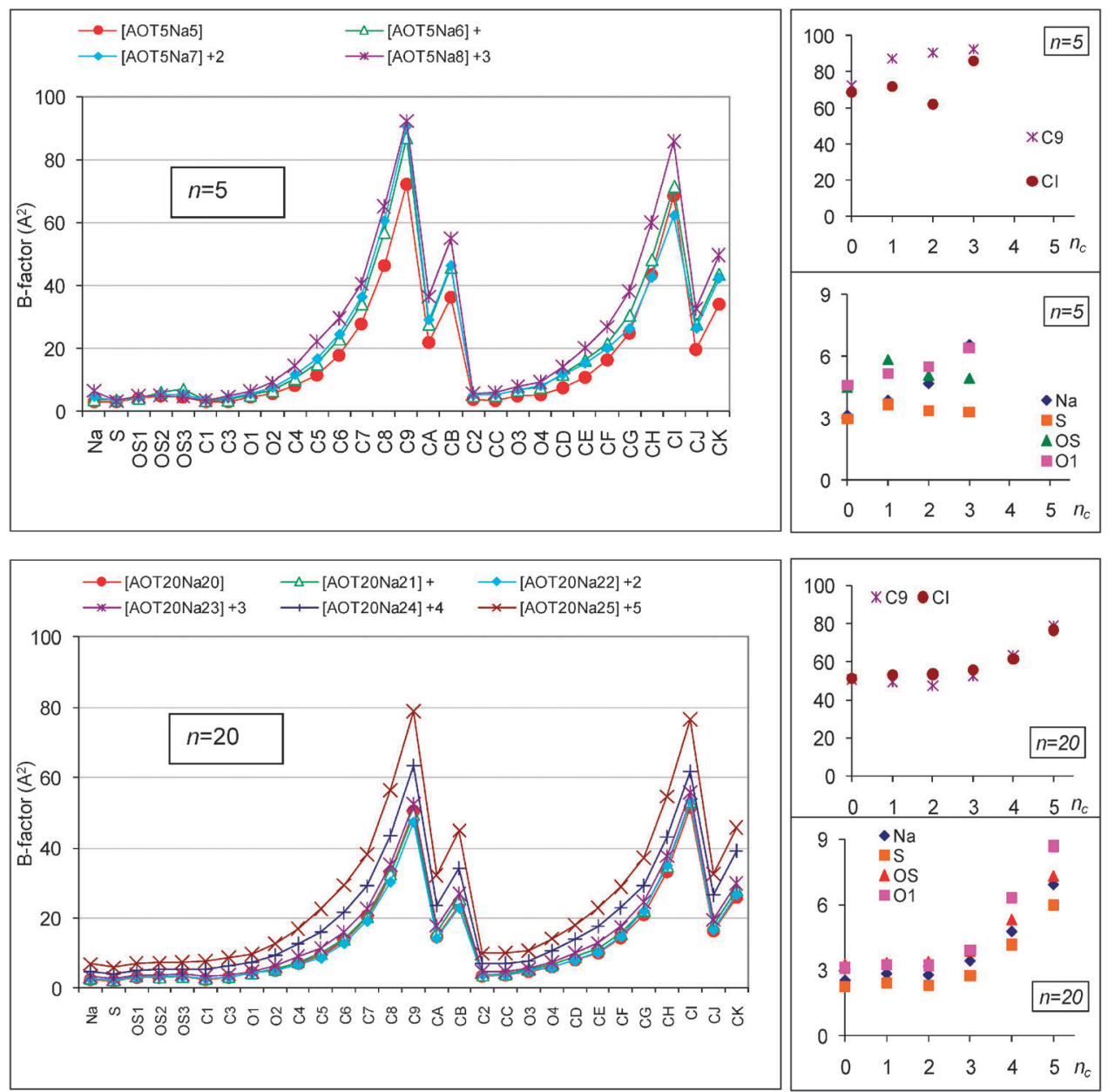

Fig. $7 B$-factor $\left(\AA^{2}\right)$ of all the atoms, labelled in Fig. 1, for $\left[\mathrm{AOT}_{5} \mathrm{Na}_{5+n_{\mathrm{c}}}\right]^{n_{\mathrm{c}}}$ and $\left[\mathrm{AOT}_{20} \mathrm{Na}_{20+n_{\mathrm{c}}}\right]^{n_{\mathrm{c}}}$ systems (left panels), and $B$-factor for some selected atoms (right panels).

Table 2 Fragmentation products, number of occurrence $(N)$ over a total of 20 independent simulations and mean fragmentation temperature ( $T$ ) for $\left[\mathrm{AOT}_{5} \mathrm{Na}_{5+n_{\mathrm{c}}}\right]^{n_{\mathrm{c}}}$ and $\left[\mathrm{AOT}_{20} \mathrm{Na}_{20+n_{\mathrm{c}}}\right]^{n_{\mathrm{c}}}$ stable aggregates $\left(i . e .\left[5^{n_{\mathrm{c}}}\right]\right.$ and $\left[20^{n_{\mathrm{c}}}\right]$ respectively)

\begin{tabular}{|c|c|c|c|c|c|c|c|c|c|}
\hline $\begin{array}{l}{\left[5^{1}\right]} \\
T=1367 \pm 60\end{array}$ & & \multicolumn{3}{|r|}{$\begin{array}{l}{\left[5^{2}\right]} \\
T=1007 \pm 70\end{array}$} & \multicolumn{5}{|c|}{$\begin{array}{l}{\left[5^{3}\right]} \\
T=609 \pm 70\end{array}$} \\
\hline $\begin{array}{l}{\left[1^{1}\right]+\left[4^{0}\right]} \\
{\left[2^{1}\right]+\left[3^{0}\right]} \\
{\left[3^{1}\right]+\left[2^{0}\right]} \\
{\left[4^{1}\right]+\left[1^{0}\right]}\end{array}$ & & $\begin{array}{l}1 \\
2 \\
9 \\
8\end{array}$ & & $\begin{array}{l}{\left[3^{1}\right]+\left[2^{0}\right]} \\
{\left[4^{1}\right]+\left[1^{1}\right]} \\
{\left[5^{1}\right]+\mathrm{Na}^{+}}\end{array}$ & & $\begin{array}{r}2 \\
16 \\
2\end{array}$ & $\begin{array}{l}{\left[3^{2}\right]} \\
{\left[4^{2}\right]} \\
{\left[5^{2}\right]}\end{array}$ & $\begin{array}{l}{\left[2^{1}\right]} \\
{\left[1^{1}\right]} \\
\mathrm{Na}^{+}\end{array}$ & $\begin{array}{r}2 \\
1 \\
17\end{array}$ \\
\hline $\begin{array}{l}{\left[20^{1}\right]} \\
T=884 \pm 60\end{array}$ & $N$ & $\begin{array}{l}{\left[20^{2}\right]} \\
T=788 \pm 50\end{array}$ & $N$ & $\begin{array}{l}{\left[20^{3}\right]} \\
T=793 \pm 70\end{array}$ & $N^{a}$ & $\begin{array}{l}{\left[20^{4}\right]} \\
T=713 \pm 110\end{array}$ & $N$ & $\begin{array}{l}{\left[20^{5}\right]} \\
T=617 \pm 80\end{array}$ & $N$ \\
\hline $\begin{array}{l}\left.8^{1}\right]+\left[12^{0}\right] \\
{\left[9^{1}\right]+\left[11^{0}\right]} \\
{\left[10^{1}\right]+\left[10^{0}\right]} \\
{\left[11^{1}\right]+\left[9^{0}\right]} \\
{\left[12^{1}\right]+\left[8^{0}\right]} \\
{\left[13^{1}\right]+\left[7^{0}\right]} \\
{\left[15^{1}\right]+\left[5^{0}\right]} \\
{\left[16^{1}\right]+\left[4^{0}\right]}\end{array}$ & $\begin{array}{l}2 \\
3 \\
2 \\
4 \\
2 \\
4 \\
1 \\
2\end{array}$ & $\begin{array}{l}{\left[10^{1}\right]+\left[10^{1}\right]} \\
{\left[11^{1}\right]+\left[9^{1}\right]} \\
{\left[12^{1}\right]+\left[8^{1}\right]} \\
{\left[13^{1}\right]+\left[7^{1}\right]} \\
{\left[14^{1}\right]+\left[6^{1}\right]}\end{array}$ & $\begin{array}{l}4 \\
8 \\
5 \\
2 \\
1\end{array}$ & $\begin{array}{l}{\left[10^{2}\right]+\left[10^{1}\right]} \\
{\left[11^{2}\right]+\left[9^{1}\right]} \\
{\left[12^{2}\right]+\left[8^{1}\right]} \\
{\left[13^{2}\right]+\left[7^{1}\right]} \\
{\left[14^{2}\right]+\left[6^{1}\right]} \\
{\left[15^{2}\right]+\left[5^{1}\right]}\end{array}$ & $\begin{array}{r}5 \\
13 \\
20 \\
15 \\
20 \\
7\end{array}$ & $\begin{array}{l}{\left[10^{2}\right]+\left[10^{2}\right]} \\
{\left[11^{2}\right]+\left[9^{2}\right]} \\
{\left[12^{2}\right]+\left[8^{2}\right]} \\
{\left[13^{3}\right]+\left[7^{1}\right]} \\
{\left[14^{3}\right]+\left[6^{1}\right]} \\
{\left[16^{3}\right]+\left[4^{1}\right]}\end{array}$ & $\begin{array}{r}3 \\
3 \\
11 \\
1 \\
1 \\
1\end{array}$ & $\begin{array}{l}{\left[13^{3}\right]+\left[7^{2}\right]} \\
{\left[14^{3}\right]+\left[6^{2}\right]} \\
{\left[15^{4}\right]+\left[5^{1}\right]} \\
{\left[10^{2}\right]+\left[7^{2}\right]+\left[3^{1}\right]}\end{array}$ & $\begin{array}{r}4 \\
13 \\
2 \\
1\end{array}$ \\
\hline
\end{tabular}

where $m_{i}$ is the mass of the atom $i$, and $\boldsymbol{R}$ and $\boldsymbol{r}_{i}$ indicate the position vectors of the aggregate centre of mass and of atom $i$, respectively. Summation was evaluated either over all atoms (total $R_{\mathrm{G}}, R_{\mathrm{GT}}$ ) or just over the core atoms, namely sodium, sulfur and oxygen atoms of the $\mathrm{SO}_{3}{ }^{-}$group (core $R_{\mathrm{G}}, R_{\mathrm{GC}}$ ). Fig. 4 shows $R_{\mathrm{GC}}$ and $R_{\mathrm{GT}}$ data for all the [AOT $\left.{ }_{n} \mathrm{Na}_{n+n_{\mathrm{c}}}\right]^{n_{\mathrm{c}}}$ systems corresponding to stable aggregates vs. $n$ and $n_{\mathrm{c}}$. A more traditional plot of the same data is provided in 
Fig. S1 of the ESI. $\dagger$ The remarkable similarity of the plot of $R_{\mathrm{GC}}$ to that of $E_{\mathrm{b}} /\left(2 n+n_{\mathrm{c}}\right)$ (Fig. 3) suggests once again the fact that the key factor for the aggregate stability stems from the electrostatic interactions of the highly charged micelle core. On the other hand, the increase of $R_{\mathrm{GT}}$ and $R_{\mathrm{GC}}$ with $n_{\mathrm{c}}$ is clearly a consequence of an increased repulsive effect due to charge addition. We may notice that the latter effect is more pronounced on $R_{\mathrm{GC}}$ than on $R_{\mathrm{GT}}$, meaning again that most of the action, so to speak, takes place at the core of the micelle.

As already observed in ref. 13, the shape of the aggregates is generally non-spherical, and is indeed quite elongated, the more so the larger the number $n$ of [AOTNa] molecules: for this reason we found convenient to approximate the shape of the aggregate by an ellipsoid. We can calculate the three principal moments of inertia $I_{1}, I_{2}$ and $I_{3}$ for each system, either for all atoms or only for the core atoms. Then, from $I_{1}$, $I_{2}$ and $I_{3}$ we considered an equivalent homogeneous ellipsoid with principal semi-axes $a, b$ and $c(a \gg b>c)$ having the same three moments of inertia. Semi-axis lengths are given by: ${ }^{13}$

$$
\begin{aligned}
& a^{2}=\left(\frac{5}{2 M_{\mathrm{tot}}}\right)\left(I_{1}+I_{2}+I_{3}-2 I_{1}\right) \\
& b^{2}=\left(\frac{5}{2 M_{\mathrm{tot}}}\right)\left(I_{1}+I_{2}+I_{3}-2 I_{2}\right) \\
& c^{2}=\left(\frac{5}{2 M_{\mathrm{tot}}}\right)\left(I_{1}+I_{2}+I_{3}-2 I_{3}\right)
\end{aligned}
$$

where $M_{\text {tot }}$ is the total mass of either the whole aggregate or just of its core.

In Fig. S2 (ESI $\dagger$ ) we report the lengths of the three semi-axes of the equivalent ellipsoid referred to all atoms (top) or to the core atoms (bottom) vs. $n$ for all $n_{\mathrm{c}}$ values corresponding to stable aggregates. These plots are hard to make use of and just allow one to conclude that $a$ is larger than $b$ and $c$ and that, above all, $a$ increases with $n$ and $n_{\mathrm{c}}$ more than $b$ and $c$ : this means that the shape of the aggregates resembles that of a cigar and this is more so with increasing number of surfactant molecules and positive ions. Clearer plots are given in Fig. 5, where $a, b, c$ for the whole aggregates and their core are plotted $v s$. $n$ and $n_{\mathrm{c}}$. While the values for $b$ and $c$ are almost constant for all systems, the $a$ values, corresponding to the length of the aggregates, show a behaviour similar to those of $E_{\mathrm{b}} /\left(2 n+n_{\mathrm{c}}\right)$ and of $R_{\mathrm{GC}} v s . n$ and $n_{\mathrm{c}}$. Again, this suggests that the stability and shape of the systems critically depend on the electrostatic forces, which, for systems of this size, produce anisotropic, almost mono-dimensional aggregates. To be more specific, we observe also that both the cores and the whole aggregates swell asymmetrically with increasing $n_{\mathrm{c}}$; this effect is more marked for the core and the increase curve is steeper for larger $n_{\mathrm{c}}$ values. Indeed, while the long semi-axis $a$ increases steadily with $n$, the short semi-axes $b$ and $c$ do not increase linearly with $n$ attaining instead almost asymptotic values $(b \approx 12 \AA$ and $c \approx 10 \AA$ for the whole aggregate, and $b \approx 6 \AA$ and $c \approx 5 \AA$ for the core). At low $n$ values the aggregates are instead quite spherical and cannot bear high excess charge without breaking (see Table 1).
In Fig. 6 we show representative MD snapshots visualising the conformations of all positively charged aggregates for $n=5\left(n_{\mathrm{c}}=1,2,3\right.$; left panels $)$ and three aggregates for $n=20\left(n_{\mathrm{c}}=1,3,5\right.$; right panels). One may clearly see that, even at high charge state, still reverse micelle-like aggregates are formed: sodium counterions and surfactant heads in the core of the micelle are arranged so that the attractive electrostatic interactions are able to overcome the repulsive ones leading to stable aggregates. The $\mathrm{Na}^{+}-\mathrm{SO}_{3}{ }^{-}$distances are quite short, so that one should consider electronic exchange forces. Obviously the latter are not explicitly considered in classical MD simulations but in our opinion this is unnecessary since ionic bonds are essentially explained by classical physics.

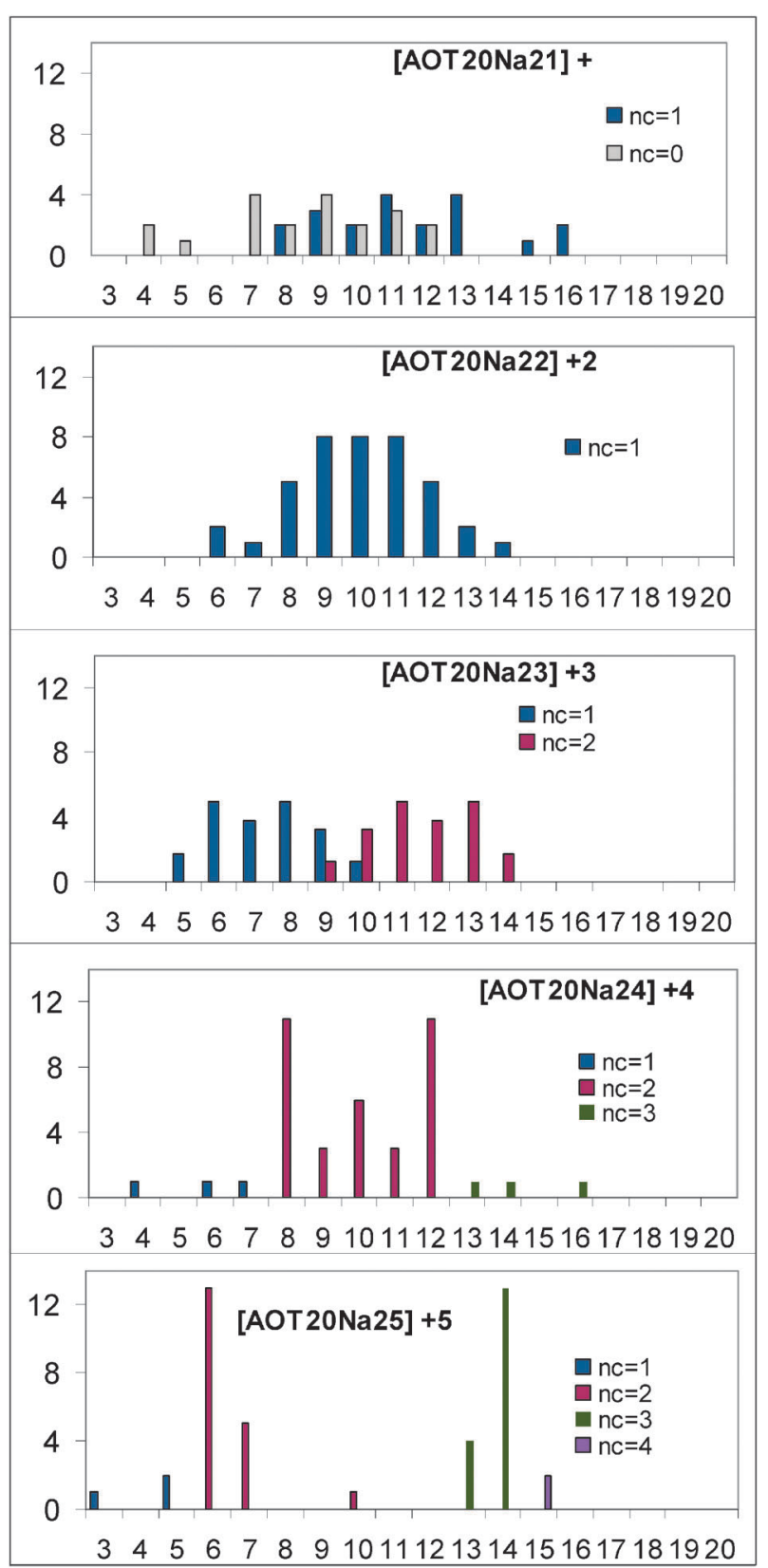

Fig. 8 Numbers of fragments $v s$. their dimension evaluated in sets of 20 independent fragmentation simulations for each $\left[\mathrm{AOT}_{20} \mathrm{Na}_{20+n_{\mathrm{c}}}\right]^{n_{\mathrm{c}}}$ system (data for $\left[\mathrm{AOT}_{20} \mathrm{Na}_{20+3}\right]^{3+}$ are averaged over 4 sets). 


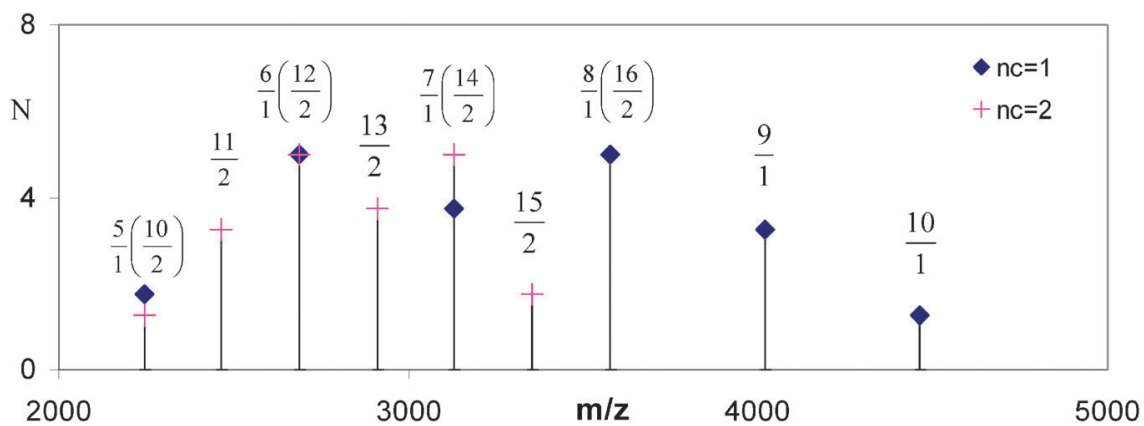

Fig. 9 Fragment abundance as a function of the mass to charge ratio, $m / z$, resulting from eighty independent fragmentation simulations of the $\left[\mathrm{AOT}_{20} \mathrm{Na}_{23}\right]^{+3}$ precursor ion (see text).

Indeed, we notice that in Fig. 2 simulation data are consistent with experimental findings.

c. Analysis of statistical atomic fluctuations. The fluctuation dynamics of the aggregates was investigated by evaluating the atomic $B$-factor defined by:

$$
B_{i}(t)=\left(\frac{8 \pi^{2}}{3}\right)\left\langle\left|\mathbf{u}_{i}(t)\right|^{2}\right\rangle
$$

where $\mathbf{u}_{i}(t)$ is the displacement of atom $i$ at time $t$, averaged over 10 ps time intervals. ${ }^{13,17}$ These values were then averaged over the last $10 \mathrm{~ns}$ of each trajectory. In the left panels of Fig. 7 the atomic $B$-factor values for the aggregates with $n=5$ and $n=20$ at various charge states are reported. It is worth to note the coexistence, within the same aggregate, of nearly motionless atoms and of atomic species characterized by relatively high mobility. This behaviour suggests that the core atoms form a quite rigid structure whereas the external layer atoms are remarkably mobile. Presumably the large fluctuations of the latter atoms may precede the aggregate fragmentation above $n_{\mathrm{c}, \max }$.

Let us now look, in the right panels of Fig. 7, at the $B$-factor values of methyl carbon atoms C9 and CI, which are far from the core (see Fig. 1). These values turn out to be larger for small aggregates than for big ones: that is to say, the larger the aggregate is, the more strictly packed the aliphatic chains are. $B$-factor values are practically independent of the charge state for low $n_{\mathrm{c}}$, but they increase steeply while approaching the aggregate stability limit. The trends for $n=5$ seem less regular simply because the $B$-factors are averaged over a smaller ensemble of atoms. It is interesting to consider the $B$ factors of the core ions for the $n=20$ systems close to the instability limit: their values turn out to be quite small $\left(3 \AA^{2}\right)$ up to $n_{\mathrm{c}}=3$ and they increase up to $9 \AA^{2}$ for $n_{\mathrm{c}}=5$.

\section{Fragmentation patterns}

We have simulated the fragmentation reactions by increasing the temperature until the selected aggregate starts breaking. Both the parent aggregate and the generated fragments are indicated here as $\left[n^{n_{c}}\right]$. In Table 2 we present the fragmentation patterns of two sets of aggregates $(n=5$ and $n=20)$ in order to evidence the effect of the aggregation number. For the $\left[\mathrm{AOT}_{20} \mathrm{Na}_{23}\right]^{3+}$ system (i.e. $\left[20^{3}\right]$ ) we can compare our results with experimental findings reported by Fang et al. ${ }^{10}$ These authors have shown that it dissociates mainly as $\left[14^{2}\right]+\left[6^{1}\right]$, $\left[13^{2}\right]+\left[7^{1}\right],\left[12^{2}\right]+\left[8^{1}\right],\left[11^{2}\right]+\left[9^{1}\right]$, and $\left[10^{2}\right]+\left[10^{1}\right]$. This fragmentation pattern closely agrees with the present data.

From Table 2 we can also conclude that neutral species generated by fragmentation of singly charged aggregates are more frequently smaller than the charged ones. Multiply charged aggregates dissociate initially as couples of lower charge state aggregates, and also in this case most frequently the generated species with low $n_{\mathrm{c}}$ are smaller in size than the generated species with higher charge excess value. A better view of this may be obtained by looking at Fig. 8, where we provide the numbers of events (represented by bars) for each fragmentation channel resulting from 20 independent MD simulations for each $\left[\mathrm{AOT}_{20} \mathrm{Na}_{20+n_{\mathrm{c}}}\right]^{n_{\mathrm{c}}}$ system, as described in the Computational methods section.

Since some pathways seem to be preferred, we report in Fig. 9 the calculated ratios of the mass of the final fragments with respect to their charge for the aggregate $\left[\mathrm{AOT}_{20} \mathrm{Na}_{23}\right]^{3+}$. These results compare satisfactorily with the experimental data reported in Fig. 4 of ref. 10 . We notice that $\left[9^{1}\right]$ and $\left[10^{1}\right]$ were undetectable in these experiments.

As for the fragmentation temperature, the trends we observe vs. $n$ and $n_{\mathrm{c}}$ (see Fig. 10) allow us to draw the following conclusions: (i) at each $n$, the fragmentation temperature decreases with $n_{\mathrm{c}}$, meaning that the aggregate stability decreases significantly with the charge state; (ii) the fragmentation temperature of singly charged aggregates $\left(n_{\mathrm{c}}=1\right)$ decreases with $n$ due to the simultaneous increase of the number of fragmentation channels, which makes the aggregate decomposition statistically more favourable; instead for $n_{\mathrm{c}}>1$ the curve for $T v s$. $n$ shows a maximum which shifts to higher $n$ with increasing $n_{\mathrm{c}}$.

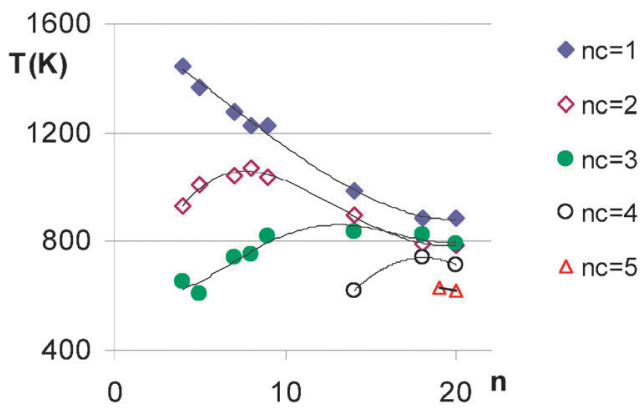

Fig. 10 Average (over twenty simulations) fragmentation temperature of charged aggregates as a function of $n$. Lines are just guides for eyes. 
This suggests that for multiply charged aggregates at low $n$ the stabilizing effect due to the increase of $n$ prevails over the abovementioned statistical effect. At high aggregation numbers,
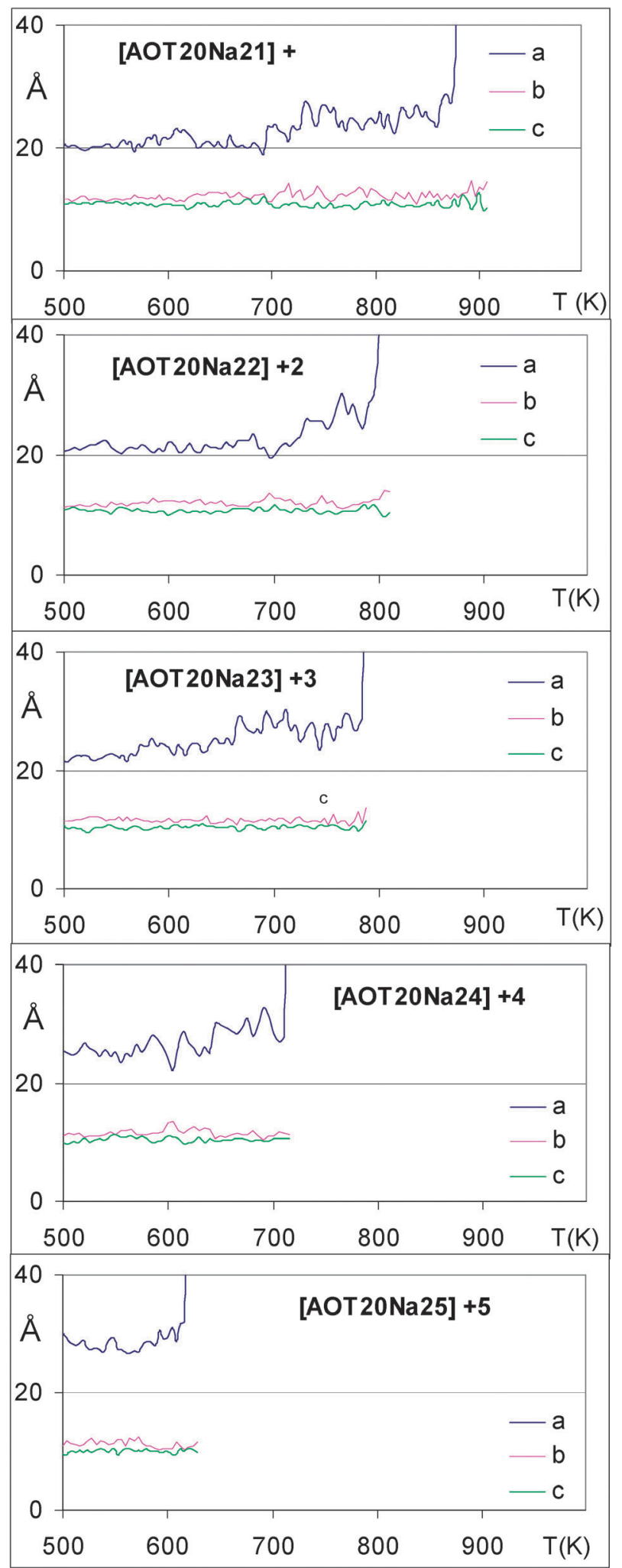

Fig. 11 Semi-axis lengths $a, b$ and $c$ of the equivalent ellipsoids $v s$. temperature for charged aggregates with $n=20$ during fragmentation simulations. the fragmentation temperature is similar for all charge states. This behaviour emphasizes a dilution effect of the extra charges in the larger aggregates.

In Fig. 11 we examine the temperature dependence of the ellipsoid semi-axis lengths $a, b, c$ during fragmentation for the cases with $n=20$. The transverse aggregate dimensions, i.e. the $b$ and $c$ values, remain practically constant, while the system gets more and more elongated, i.e. $a$ (the length of the longest semi-axis of the ellipsoid) is subject to large fluctuations, with a sudden increase close to the breakup of the system.

\section{Conclusions}

In this work, for the first time at the best of our knowledge, MD simulations are reported providing equilibrium properties of positively charged supramolecular [AOTNa] aggregates formed in vacuo at various aggregation numbers $(n=1-20)$ and various positive charge states $\left(n_{\mathrm{c}}=0-5\right)$. We have also performed fragmentation MD simulations for a number of the above aggregates.

Structural and dynamical features of these supramolecular aggregates appear to be largely dominated by the electrostatic interactions between sodium counterions and negatively charged surfactant heads, in agreement with previous results. ${ }^{13}$ We can also draw the following conclusions: (i) irrespective of the charge state, all the investigated aggregates show a reverse micelle-like structure, i.e. an internal charged core formed by sodium counterions and surfactant head groups surrounded by surfactant alkyl chains; (ii) the maximum number of extra charges which can be safely accommodated by an aggregate increases with $n$ in a non-linear fashion for low $n$ values; (iii) by increasing the charge state, the aggregates become more oblate and their stability decreases steeply; (iv) while core atoms are nearly motionless as in a solid-like state, peripheral atoms display a higher mobility; moreover, alkyl chains are more strictly packed in larger aggregates than in smaller ones, and at the highest possible $n_{\mathrm{c}}$ values, atomic fluctuations are significantly enhanced; (v) the fragmentation temperature of singly charged aggregates decreases with $n$, while multiply charged aggregates show a characteristic trend with a maximum, presumably resulting from the balance of electrostatic interactions and the availability of multiple fragmentation pathways; (vi) from a more general perspective, the quite interesting ability of molecular AOT-Na clusters to host an excess of charges and still be stable appears to be due mainly to the simultaneous presence of positive and negative charges and to their peculiar spatial distribution.

\section{Acknowledgements}

Financial support from MIUR 60\% and Fondazione Cariplo is gratefully acknowledged.

\section{References}

1 R. Dong and J. Hao, Chem. Rev., 2010, 110, 4978-5022.

2 K. Binnemans and C. Görller-Walrand, Chem. Rev., 2002, 102, $2303-2346$. 
3 P. L. Luisi, L. J. Magid and J. H. Fendler, Crit. Rev. Biochem., 1986, 20, 409-474.

4 G. Siuzdak and B. Bothner, Angew. Chem., Int. Ed. Engl., 1995, 34, 2053-2055.

5 D. Nohara, T. Ohkoshi and T. Sakai, Rapid Commun. Mass Spectrom., 1998, 12, 1933-1935.

6 D. Nohara and M. Bitoh, J. Mass Spectrom., 2000, 35, 1434 1437.

7 C. L. Hanson, L. L. Ilag, J. Malo, D. M. Hatters, J. G. Howlett and C. V. Robinson, Biophys. J., 2003, 85, 3802-3812.

8 D. Bongiorno, L. Ceraulo, A. Ruggirello, V. Turco Liveri, E. Basso, R. Seraglia and P. Traldi, J. Mass Spectrom., 2005, 40, $1618-1625$.

9 M. Sharon, L. L. Ilag and C. V. Robinson, J. Am. Chem. Soc., 2007, 129, 8740-8746.

10 Y. Fang, A. Bennett and J. Liu, Int. J. Mass Spectrom., 2010, 293, $12-22$.

11 F. Cacace, G. de Petris, E. Giglio, F. Punzo and A. Troiani, Chem.-Eur. J., 2002, 8, 1925-1933.
12 G. Giorgi, L. Ceraulo and V. Turco Liveri, J. Phys. Chem. B, 2008, 112, 1376-1382.

13 G. Longhi, S. L. Fornili, V. Turco Liveri, S. Abbate, D. Rebeccani, L. Ceraulo and F. Gangemi, Phys. Chem. Chem. Phys., 2010, 12, 4694-4703.

14 J. Wang, R. M. Wolf, J. W. Caldwell, P. A. Kollman and D. A. Case, J. Comput. Chem., 2004, 25, 1157-1174.

15 C. I. Bayly, P. Cieplak, W. Cornell and P. A. Kollman, J. Phys. Chem., 1993, 97, 10269-10280.

16 D. A. Case, et al., AMBER 10, University of California, San Francisco, 2008.

17 M. P. Allen and T. J. Tildesley, Computer Simulation of Liquid, Clarendon Press, Oxford, 1987.

18 W. Humphrey, A. Dalke and K. Schulten, J. Mol. Graphics, 1996, 14, 33-38.

19 D. L. Bergman, L. Laaksonen and A. Laaksonen, J. Mol. Graphics Modell., 1997, 15, 301-306.

20 R. A. Sayle and E. J. Milner-Whilte, Trends Biochem. Sci., 1995, 20, 374-376. 